\title{
A FORMALISATION APPROACH FOR COLLABORATIVE USER EXPERIENCE DESIGN
}

\author{
D. Kerpen ${ }^{凶}$, J. Conrad and D. Wallach \\ Hochschule Kaiserslautern, Germany \\ $\triangle$ daniel.kerpen@hs-kl.de
}

\begin{abstract}
We propose to combine Collaborative User Experience Design (CUXD) projects with the integrated product and process modelling theory CPM/PDD to formalise the CUXD process model. CPM/PDD is discussed as a Design Theory and Methodology (DTM) to describe a product as well as the product development process based on a clear distinction of characteristics and properties. CUXD is presented as a cross-disciplinary, human-centred development model. It focuses on team collaboration, relates to concepts of Design Thinking, Agile Development as well as Lean UX and it highlights user experience metrics.
\end{abstract}

Keywords: collaborative design, product development, design models, human-centred design

\section{Introduction}

This contribution starts from the premise of an evident need to pool digitization competencies in engineering: Such an endeavour is mandatory to support the transformation of traditional products and services towards networked/smart products and innovative interactive services (see e.g. Eigner et al., 2016). We consider the picture to be only complete when acknowledging the interdependent and recursive relationship between engineering, innovations and their respective environments (e.g. social and economic aspects of innovation contexts) in such "integrated", digitized engineering lifecycles (Tafvizi Zavareh et al., 2018). In times of dynamic and radical change with increased innovation pressure, we consider it first and foremost important to highlight the relation between digitized engineering and innovative design methods to support operational and strategical success of engineering organisations in product/service development.

\subsection{Relation between digitized engineering and innovative design methods}

On the one hand, this calls for design methods in product/service development that demonstrate their innovative potential in being flexible enough to tackle complex and open-ended problems. As Dorst (2011) states, individuals associated with finding possible solutions to such problems need "to figure out 'what' to create, while there is no known or chosen 'working principle' that we can trust to lead to the aspired value" (Dorst, 2011, p. 524). With reference to Schön's (1983) idea of professional "reflective practice" in order to understand complex organisational/social problems as well as drawing on Whitbeck's (1998) idea of a responsible, ethics-based conduct of engineering, Dorst (2011) proposes a concept of "design reasoning" in form of "productive" or "open" reasoning (ibid., p. 524f.). His discussion points at open questions of his approach when calling out 
for the need to articulate these design practices in greater detail (Dorst, 2011, p. 531). On the other hand, we consider that design practices need to demonstrate their organisational impact in relation to an entrepreneurial point of view. This then calls out for design approaches which need to be standardisable and quantifiable (Wallach et al., 2017). From a business and management point of view, such 'measurable' design approaches/methods allow to make a significant contribution to operational and strategical success when compared to traditional methods of product development (Luedeke et al., 2018; Tullis and Albert, 2013; Wallach et al., 2017).

\subsection{Scope of this contribution}

We propose a concept on how to approach Collaborative User Experience Design (CUXD) projects in an iterative and standardisable manner by refering to Characteristics-Properties Modelling/Property-Driven Development (CPM/PDD). This contribution is, first and foremost, a conceptual combination of the two frameworks CUXD and CPM/PDD. Nevertheless, it promotes a valid basis for discussing the introductorily described nexus of digitized engineering and design methods. In order to do so, we start with a problem analysis (section 2) which reviews the development of (interactive) products and services from a mechanical engineering and engineering design viewpoint. We highlight the field of design theory and methodology (DTM, section 2.1) as an important reservoir for efforts striving to develop integrated frameworks. Such a perspective is described by CPM/PDD (section 2.2). Throughout this contribution, we emphasize a human-centered perspective by exemplifying how to take user experience (UX) in collaborative ways into account and how to make UX quantifiable by metrics (section 2.3). By doing so, we pave the way for describing the CUXD process (section 3) and finally map the central characteristics and domains of both concepts, CUXD and CPM/PDD (section 4) to highlight core overlaps and contact points. In this sense - while not being without limitations - the joined CUXD-CPM/PDD process model supports the continuing task of establishing a common set of design approaches. This is especially important in context of engineering project groups which increasingly include experts from various professional/interdisciplinary backgrounds. We conclude by hinting at possible application areas in mechanical engineering and industrial applications (section 5) which offers additional starting points for further research, respectively.

\section{Problem analysis}

\subsection{A vast field with heterogenous models existing in parallel: Design theory and methodology (DTM)}

Weber (2014) presents a comprehensive overview of DTM. Based on scientific findings, DTMs objective is to determine how much designing can be systemized and automated as well as to develop concepts that make the activity teachable and trainable (Conrad et al., 2018).

DTM combines a large number of different approaches, with some of them - not surprisingly being incompatible with each other. In the variety of approaches available, some have emerged as particularly popular during the last decades since their formulation in the 1980s/1990s (Conrad et al., 2018): For instance, John Gero's (1990) "function behaviour structure model" as an approach from the research field of artificial intelligence (AI), whereas Suh (1990) introduced a model called "Axiomatic Design" that basically describes the design process as a mapping from a functional space to a physical space. Following Conrad et al. (2018), the VDI guideline 2221 (VDI, 1987) and the fundamental works of Pahl and Beitz (2007) play an essential role as a general framework and summary for design guidelines in Europe and especially in Germanspeaking countries.

With several models existing in parallel, an integrating framework is considered as being beneficial (cf. Weber, 2014, p. 328). Such an integrating endeavour is presented by the approach of modelling products and development processes based on product characteristics and properties, called CPM/PDD (Characteristics-Properties Modelling, Property-Driven Development). 


\subsection{DTM Complexity calls for an integrated framework: Characteristics- properties modelling/property-driven development (CPM/PDD)}

In this section, we briefly explain the Characteristics-Properties Modelling/Property-Driven Development (CPM/PDD) approach by relying on and summing up of previous work by Conrad et al. $(2008,2018)$. We will first highlight our notion of CPM/PDD as an approach incorporating both strands of a more analytical product model domain on one side together with the more synthesis-oriented domain that allows for incremental and iterative changes of features or additions in the product development process. After outlining CPM/PDD subsequently, we will explore how to use this notion of dual analysis/synthesis characteristics for applying CPM/PDD to a procedure and its accompanying methods, finally resulting in a close collaboration-oriented and user experience-oriented agile approach.

The most significant feature of the Characteristics-Properties Modelling/Property-Driven Development (CPM/PDD) approach is the differentiation between characteristics and properties of a product (Conrad et al., 2018): Characteristics (C) cover all the items of a product that can be directly determined and influenced by a designer. These include, for example, the geometry, structure, shape, spatial configuration and material consistency. Properties (P) describe "a product's behaviour" and cannot be directly determined by a designer (e.g. weight, safety, aesthetic properties, usability), but indirectly influenced through modifications of characteristics.

The links between characteristics and properties are represented by relations. These can be read in two directions: in the analysis direction $(\mathrm{R})$, characteristics are known and the product's properties are derived (Figure 1 left). In the synthesis direction $\left(\mathrm{R}^{\wedge}-1\right)$, properties are known/required and the product's characteristics are established (Figure 1 right). In addition, Dependencies (D) respect potential constraints on the characteristic side and External Conditions (EC) represent the context in which such relational statements are valid.
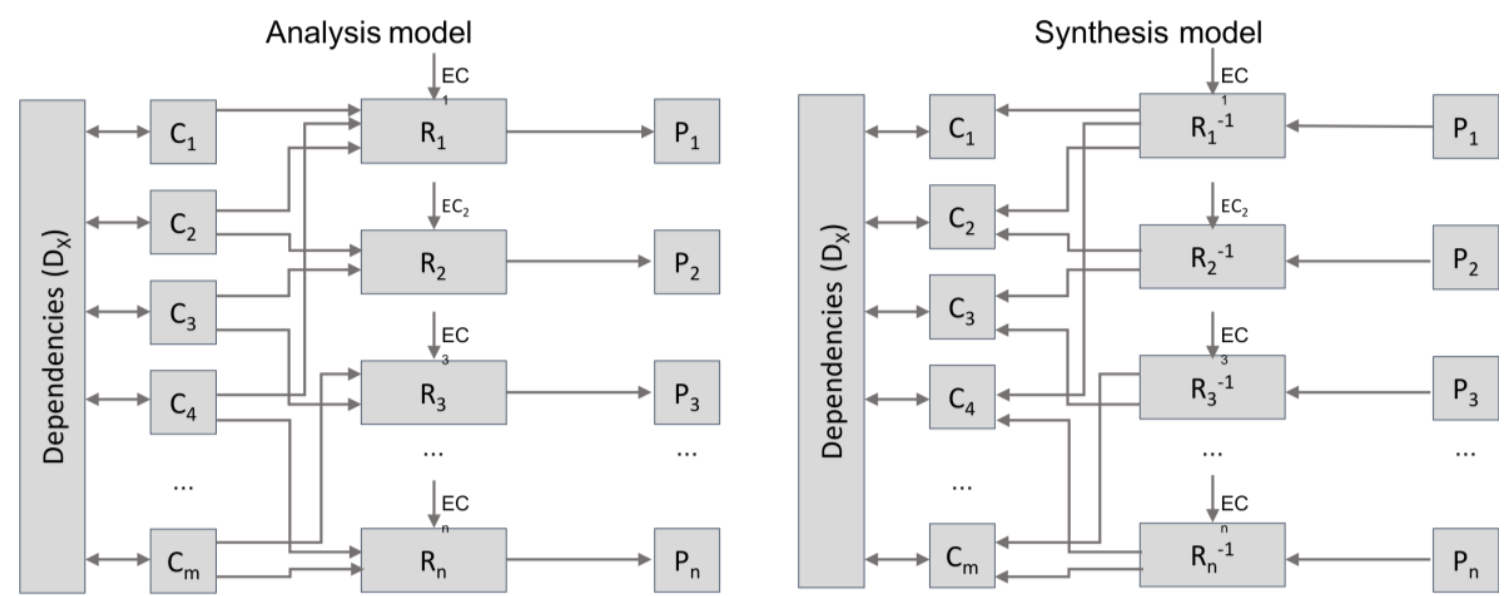

Figure 1. Differentiation between analysis and synthesis model

(Weber, 2005; Conrad et al., 2008, 2018)

The modelling of both, the product and the process is a major advantage of the CPM/PDD compared to models mostly focussing on the process model. Among other things, this simplifies the development of products, where requirements are usually not directly realizable (if users are particularly addressed, see below). Especially, CPM/PDD provides a clear framework for evaluation and process control due to its strong focus on analytical rigor: "based on known/given characteristics (structural parameters, design parameters) of a product, its properties are determined (and therefore, its behaviour), or - if the product does not yet exist - predicted" (Weber, 2014, p. 332). With a focus tending more to synthesis, the task of a development engineer/designer is to find appropriate solutions, i.e. putting together an appropriate set of characteristics that meet a (prospective) users' requirements satisfactorily.

Conrad et al. (2018) illustrate how methods from user-centered design (UCD) contribute to making concrete relations by the identification and evaluation of suitable user requirements. Starting from 
such a perspective on user requirements, we further expand the reach of requirements in the next section by taking the broader perspective of stakeholders and time and flexibility demands.

\subsection{Users, tasks, and environments revisited: Stakeholders' requirements, design thinking, and agile development}

The referenced ISO 9241-210 serves as the basis for many UCD methods aiming at products and services being usable, manageable, efficient and effective by realizing relevant user requirements. Based upon an explicit understanding of users, tasks, and environments to go through during systems' design, the ISO 9241-210 proposes four phases, i.e.:

1. understanding and specifying the context of use,

2. specifying the user requirements,

3. producing design solutions, and

4. evaluating the design.

By emphasizing these phases, design solutions are expected to result which comprehensively address the user experience and which have a strong notion of commitment to and refinery by user-centered evaluation.

Despite UCDs appropriateness in many use cases and application areas, we prefer to use the term human-centered in the remainder of this contribution: in successful development projects, the sometimes-contradictory requirements of the various stakeholders involved must be carefully balanced against each other. An exclusive focus on user needs without appropriate consideration of technical framework conditions or (justified) business goals of managerial addresses might hinder a sustainable product success. In this context, we refer to a development process as human-centered if it is based on iterative validations of a product concept with continuous involvement of users, and where the results are shared and discussed by various stakeholders within a development team (Steimle and Wallach, 2018). Or to put it short: human-centeredness focuses on the needs of the people in the context of "feasibility", "desirability" and "viability" (Luedeke et al., 2018).

Luedeke et al. (2018) pinpoint design thinking (DT) as another central approach focussing on user needs; following five iterative modes, DT strives to:

1. empathize insights by focussing on human behaviour and everyday life by using (scientifically sound) methods such as observations and interviews,

2. define all these empathy findings summarized in a point of view focused on specific users,

3. ideate the exploration of a wide variety of possible solutions using iterative ideation methods,

4. prototype idealized solutions by tangible objects, and

5. test (refined) solutions while continually improving the design (Luedeke et al., 2018; d.school, 2013).

Having briefly summarized human-centeredness and DT, we need to consider time as an important constraint - and flexibility as an answer to tackle this constraint. This consideration leads to agile methods that enable organisations to react more flexibly to the changing requirements in development domains, such as software development (Steimle and Wallach, 2018): Human-Centeredness, incorporating people's needs into new methods of product development, seems to be ideally suited for agile project management methods. In this way, human needs can be incorporated into agile processes as requirements and subsequently worked out in incremental development steps. In order to successfully manage large, i.e. extensive and demanding, projects, agile development uses the simple trick to divide a large project into several small projects (sprints), lasting only a few weeks, i.e. between two and four weeks typically. In each sprint, a "piece" (increment) of the development project (typically: software) is completely implemented so that it is available in executable form. Examples of agile methods comprise e.g. "Scrum", "Extreme Programming", or "Crystal" (Haberfellner et al., 2015).

UX metrics provide crucial directions for designing interactive systems. Metrics for measuring user experience comprise criteria that refer to actual usage situations like the effectiveness or efficiency of a system. Such metrics, as results of measurements, are quantitative by nature: for instance, completion rates or error rates can be used to operationalize the effectiveness of a system, while 
time on task (i.e. defined as the time resources used in relation to the results) is an example for a quantification of the efficiency criterion. Efficiency is without doubt one of the most important metrics when judging the performance of an interactive system (Wallach et al., 2017).

By having summarized human-centeredness, DT, and agile methods, we have a common ground to conclude the related work in order to have a closer look at Collaborative User Experience Design (CUXD) in section 3 and synthesizing CUXD and CPM/PDD in section 4.

\section{CUXD outline and process description}

\subsection{CUXD outline}

Collaborative User Experience Design (CUXD) is a process model for UX design proposed by Steimle and Wallach (2018). CUXD is targeted at the design of product with a clear focus on defining the entire interaction of a user with a product or service. CUXD is a collaborative approach, i.e. products or services are developed by a cross-functional team. It is rooted in Design Thinking and Lean UX and represents an agile, hypothesis-based approach. The CUXD model divides the development process into the three phases of "Understanding", "Exploration", and "Realization", each consisting of different workshops with suggested methods (see Figure 2).

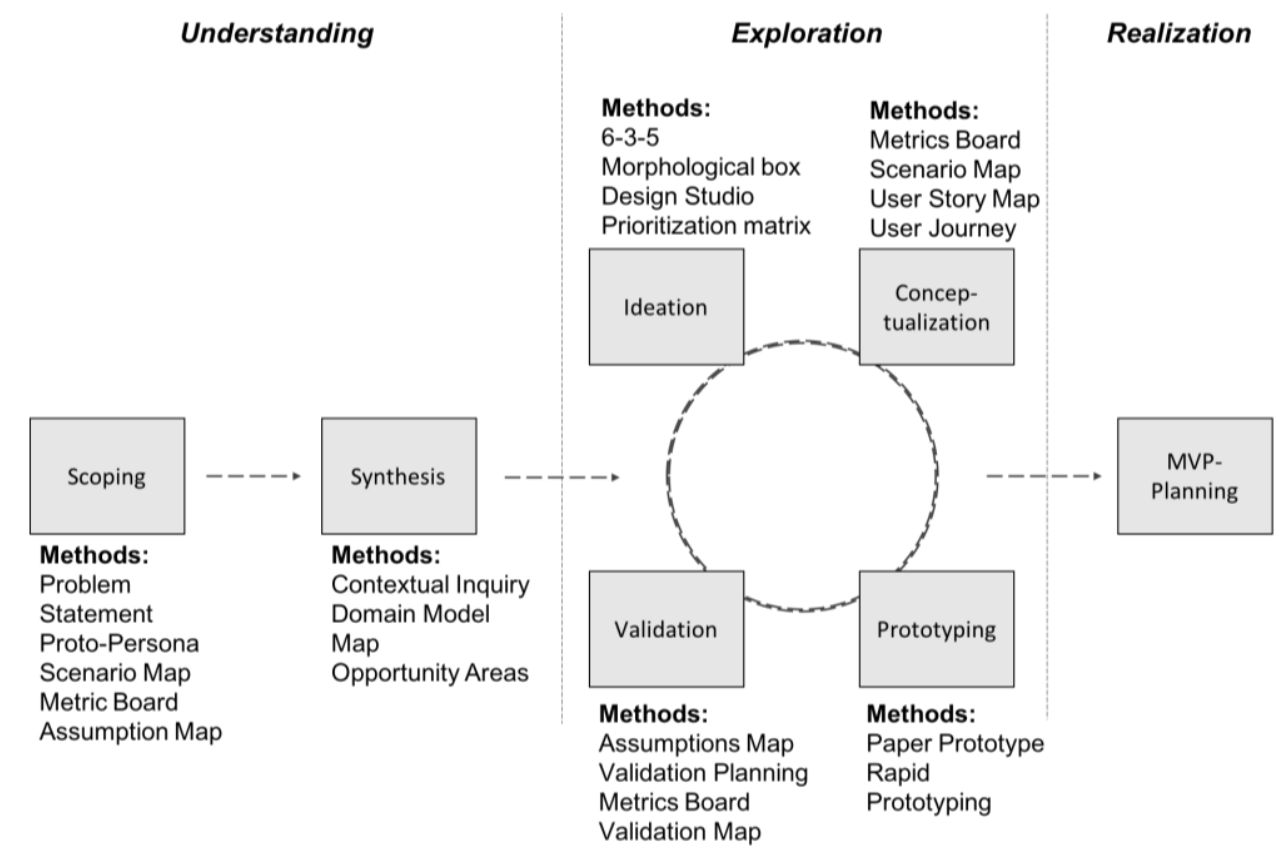

Figure 2. Collaborative UX design-process and methods (Steimle and Wallach, 2018)

The conception and development of software increasingly take place in cross-disciplinary teams (Steimle and Wallach, 2018). Individual members of such a team contribute bring expertise in various areas product management, marketing, UX design, implementation, testing, and operation - to the table. By working together over a longer period of time, such interdisciplinary teams conceive and design a product/service in joint workshops that build on each other. These workshops refer to the following individual core issues: scoping, synthesis, ideation, conceptualization, prototyping, validation, and MVPplanning. Workshops are useful formats because they allow clear guidance of the process steps and offer the possibility of flexible project planning depending on the availability of team members.

\subsection{CUXD process description}

\subsection{1. "Understanding" the issue, context(s), and assumptions}

The Scoping workshop format follows the goal to get a design project up-and-running. By drawing on problem statements or scenario maps, a team sharpens the mission and objectives, e.g. the core of the 
project order together with the client. Team members work out the status quo of market companions' products and characterize their peculiarities, advantages, and disadvantages. An essential goal of scoping workshops is to uncover the - often implicit - assumptions behind project missions, for example, to substantiate existing hypotheses about the (prospective) users of an application. In sum, critical assumptions are identified and research measures are selected for verification within the scoping workshop, after which the defined research measures are tackled.

In the Synthesis workshop, the members of a team evaluate the results of research activities. For this purpose, descriptions of existing workflows are created and product opportunities for their optimization are identified. Such analyses allow the verification of assumptions about users and support the formulation of empirically founded personas as archetypically modelled user representatives. At this point, a reflection of the previously defined project order is important: is it still compatible with the new state of knowledge or do corrections have to be made?

The two workshops outlined so far - Scoping and Synthesis - are primarily concerned with gaining a comprehensive understanding of the problem/issue, its context(s), and underlying assumptions: hypotheses are formed, data collected and hypotheses validated. This serves as a solid starting point to explore possible solutions in subsequent workshops.

\subsection{2. "Exploration" of possible solutions}

During the Ideation workshop, the team rallies to search for solution ideas to identified product opportunities. In the workshop, different creativity methods are used for the mutual inspiration of the team members (e.g. group-structured brainwriting using the 6-3-5 method): the aim of the workshop is to generate as many ideas as possible. The result of the Ideation workshop is a prioritized idea catalogue for product design.

In the Conceptualization workshop, the previously developed idea catalogue is formed into a coherent solution. Hereby, team members develop a vision of possible usage scenarios and - on this basis derive a picture of future functionalities of a solution: in this workshop, a first and abstract (high-level) view of the future user interface of a product is created. The abstract concept of the user interface is successively concretized until finally a concept proposal for the product is available. As a result of the workshop, a user journey can be created, illustrating user interactions through a series of visualized screens in order to achieve work goals.

It is important to note that the user journey again conceals assumptions: assumptions about the suitability of certain solution approaches to fulfil user needs. The identification of these assumptions is the subject of a Prototyping workshop, their validation is the focus of the subsequent Validation workshop. For this purpose, a validation plan is derived which describes the type of prototype required to validate the concept. Afterwards, work begins on the joint development of a prototype. The aim here is not to specify a solution, but primarily to prepare a concept review. After the Prototyping workshop, developed prototypes are finalized and tested. For example, it is possible to observe users interacting with a prototype and to incorporate their feedback into the further development of evolved concepts.

The results of these observations are evaluated in the Validation workshop. Members of a team compile recorded observations, categorize and weight them. During validation, reference is continuously made to the originally defined assumptions, and it is evaluated whether the developed concept is based on a sufficiently resilient foundation. If this evaluation supports the concept, then its implementation in a first small release and the subsequent evaluation of market feedback should be the obvious choice.

Often, a Validation workshop opens up various detailed issues that lead to revisions of a prototype. A fundamental revision of the concept might also become necessary and thus require further ideation workshops. Hence, in this phase, iterations between workshops are just as much a part of a typical project as far-reaching modifications of prototypes.

\subsection{3. "Realization" in a lean way}

If, on the other hand, a concept proves its worth, the aim of the last workshop - MVP-Planning - is to define a first minimum version of a convincing release, a Minimum Viable Product. For this purpose, the relevant functionalities of a product are prioritized again in the team. Various factors, such as the expected benefit for users and customers, the contribution to business goals or the costs of 
implementation need to be considered. A roadmap to define future enhancements is defined and concrete quantitative metrics to measure a product's/service's success (or the lack of it) using a metric board (e.g. Wallach et al., 2017) are established.

These seven central workshops taken together form CUXD as a process model that is theoretically based on the cornerstones of human-centeredness, design thinking, agile development, and Lean UX. Let us shortly focus on the latter one, Lean UX (Gothelf and Seiden, 2012), with its notion of solving time constraints through increased flexibility. Consider the image of an interdisciplinary team with members working together over a certain period of time and sharing a joint (physical) workspace; a team in which, ideally, every stakeholder is involved in each phase. Roles should have as little significance as possible, no matter whether team members are developers, designers or product managers. This consideration points to the advantages of a cross-functional cooperation: the entire know-how of the team is incorporated into the product design. Problems that would otherwise only occur by information - or even only chance - discovered during commissioning of the final product can be disclosed at an early stage. This avoids high revision costs and misinvestments. Furthermore, the effort associated with comprehensive specification documents can be drastically reduced because long specification documents are replaced by descriptive prototypes. Members of the involved team can identify themselves with a product concept, all team members are responsible for the success of the product - an attribute that CUXD inherits from Lean UX.

In a nutshell, at CUXD's core, a defined process is iteratively repeated: the team selects a relevant question, asks users about it, begins to develop an optimized solution concept, visualizes and validates it with users, and, if necessary, quickly adapts and implements it and evaluates the result.

\section{CUXD and CPM/PDD synthesis}

In this section, CUXD is combined with the product development methodology CPM/PDD in order to utilize the findings and advantages of the previous sections. For this purpose, each phase of CUXD is considered separately and compared with the contents of the CPM product model and the process steps of PDD.

\section{1. "Understanding"-phase in CPM/PDD}

The first phase "Understanding" with its workshops Scoping and Synthesis are preparatory steps that are not addressed directly in the CPM/PDD model. Nevertheless, fundamental and necessary components of the model are created and defined here. In particular, a result of these workshops is a quantity of the product's required properties (PR). Through the way of determination (e.g. through Problem Statements, Proto-Personas or Journey Maps), these are determined in a user-centered manner to promise a good matching with the "real" user requirements (see section 2.3). An important side effect of this phase is the determination of External Conditions (EC), i.e. the framework conditions under which the later relations ( $R$ or $\mathrm{R}^{\wedge}-1$ ) are valid. The left side of Figure 3 summarizes the outcomes of the first phase.
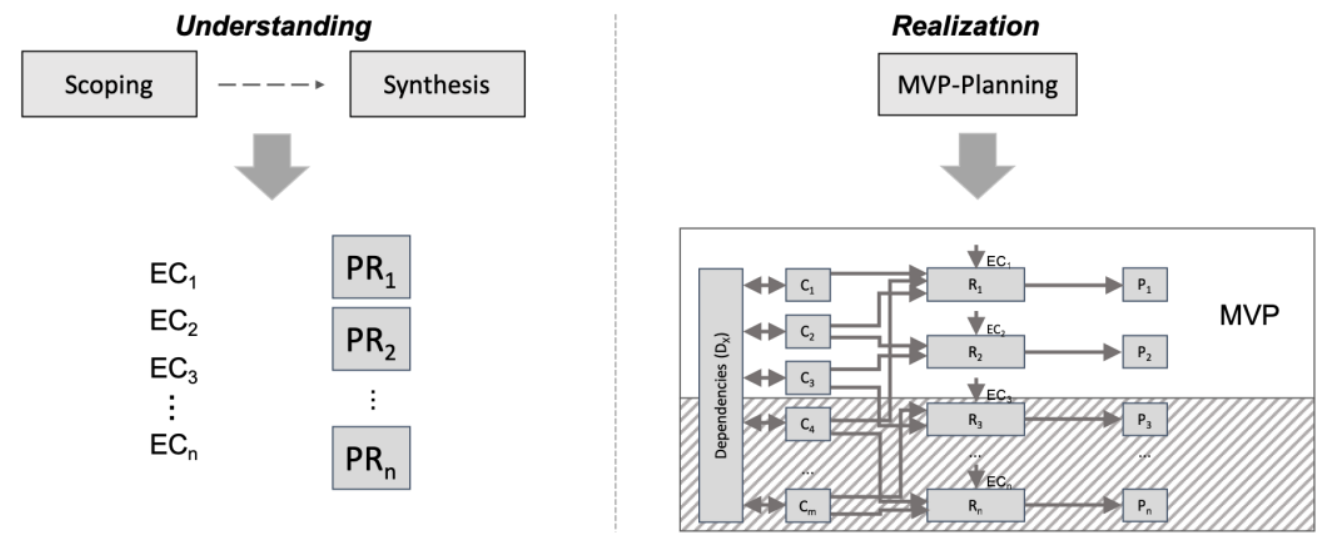

Figure 3. "Understanding"-phase (left) and "Realization"-phase (right) and CPM/PDD (based on Weber, 2005; Conrad et al., 2008, 2018) 


\section{2. "Exploration"-phase in CPM/PDD}

The "Exploration"-phase is an iterative sequence of ideation, conceptualization, prototyping, and validation (see Figure 4).
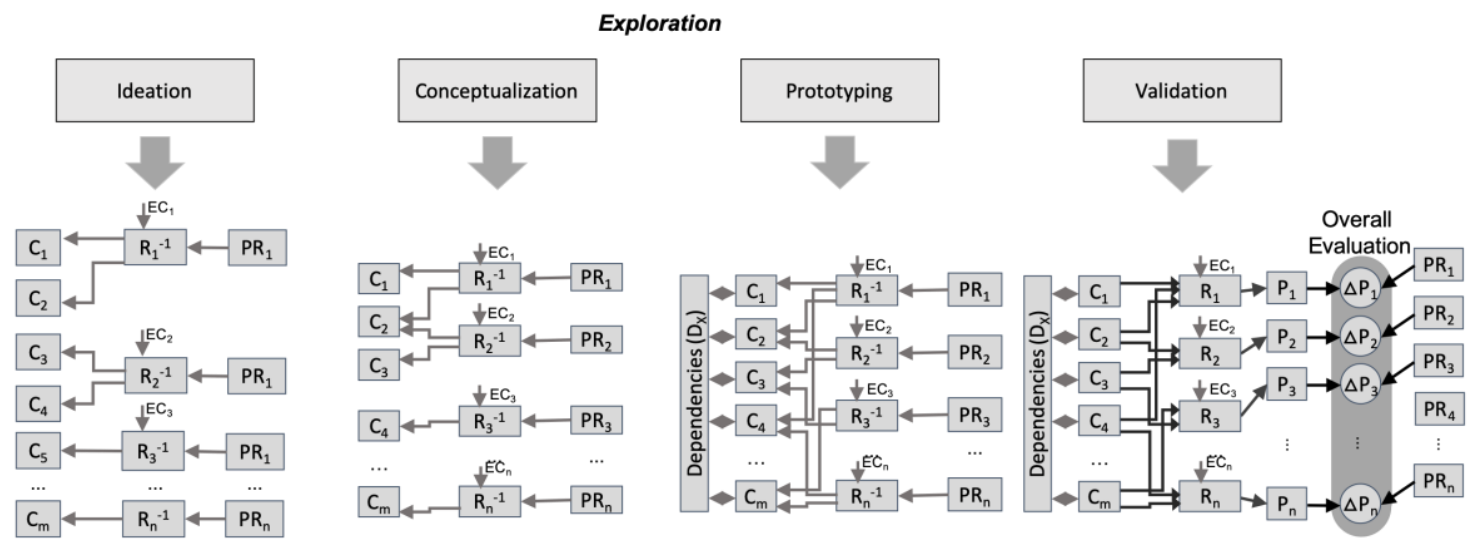

Figure 4. "Exploration"-phase and CPM/PDD

(based on Weber, 2005; Conrad et al., 2008, 2018)

Ideation is supposed to generate as many ideas as possible for the identified user requirements. The user requirements are mostly considered individually and translated into features. The synthesis methods used (see Figure 2) are rather informal and strongly influenced by intuition and creativity. The evaluation of the developed solutions is also largely based on common sense. For CPM/PDD, this means that there is a multitude of different CPM product models based on individual required properties (PR). The synthesis relations $\left(\mathrm{R}^{\wedge}-1\right)$ are not formalised but largely based on the abilities of the people involved.

In the Conceptualization step, the results of previous activities are more formalised and fewer different solutions are considered. In order to obtain results that completely fulfil the problem, the user requirements are more strongly integrated. For the considerations in CPM/PDD, this means that there are fewer different CPM product models, but these fulfil already several required properties PR. The formalisation of synthesis relations is also increasing.

Prototyping produces the first tangible results. It focuses on one or very few variants that already integrate many of the user requirements. The range of these prototypes extends from low- to highfidelity. Typical techniques for low-fidelity prototypes are paper prototypes or pencil scribbles. This is particularly useful in the early phases of prototyping so that these can be discarded easily and without emotional reference. In later iterations, the prototypes become more and more sophisticated, e.g. clickable user interfaces or 3D printing. For the CPM model, this implies that (almost) all PR are considered in the analysis step. Especially for sophisticated prototypes, the synthesis relations become more and more complex, also CAD models or source code are already required.

The Validation workshops determine the fulfilment of the user requirements by the prototype. Not only the fulfilment of individual requirements is relevant for the development process, but also the consideration of an overall evaluation. For CPM/PDD the analysis of the submitted solution is in focus: the individual properties are evaluated with regard to their target fulfilment. But especially the overall evaluation of the solution is particularly interesting as it determines the next steps. If the overall evaluation is sufficient for the problem statement, the next phase can begin.

\section{3. "Realization"-phase in CPM/PDD}

In the "Realization"-phase, a product is — partially — reified. In the sense of the MVP and the lean approach, it is only intended to implement a part of the most important requirements. This fact can be delineated very clearly in the CPM model since it becomes visible which characteristics are necessary for the implementation of the MVP (see Figure 3 on the left side); however, the possibilities and limitations of MVPs in mechanical engineering is a particular topic that should be considered separately in research and practice. 


\section{Conclusion}

In this contribution, we have shown that it is possible to formalise the lean and agile CUXD process approach (abbreviated for Collaborative User Experience Design) through the combination with Characteristics-Properties Modelling/Property-Driven Development (CPM/PDD). CUXD is a crossdisciplinary, human-centered development model focussing on collaboration in teams. CPM/PDD is a methodology to describe a product as well as the product development process based on the clear distinction of characteristics and properties.

This contribution is, first and foremost, a conceptual combination of the two frameworks CUXD and CPM/PDD. We identified relevant touch points of the two approaches and we pointed out on how CPM/PDD can serve as a basis for the formalisation of CUXD. By doing so, we promote a valid basis for discussing the introductorily described nexus of digitized engineering and design methods. In this sense, the joined CUXD-CPM/PDD process model supports the continuing task of establishing a common set of design approaches.

Such formalisation is necessary to make the whole approach of developing (interactive) products and services quantifiable and trainable/teachable. This is especially important in context of engineering project groups which increasingly include experts with various professional/educational backgrounds. We suggest that the CUXD and CPM/PDD combination can be particularly helpful for at least:

1. the determination of the maturity level of a current development process,

2. supporting the internal organisational communication and co-operation, especially when considering development through cross-functional teams, and

3. being compatible to the inclusion of digital tools which are important means of support in everyday work life.

From an industry application perspective, conceptual approaches cannot be seen without limitations. Therefore, future work has to illustrate the value of the contribution by using case studies from the engineering lifecycle to present the broad applicability of the concept. For instance, and with regard to the points 1-3 listed above, we plan to support and to evaluate the development process by using digital tools for recording the product features and properties in the individual steps of the CUXD. On this basis, synthesis and analysis relations can be accompanied digitally; furthermore, collaboration can be realized by distributed teams working jointly together via remote collaboration tools in different research/design labs.

\section{Acknowledgement}

We gratefully acknowledge constructive feedback of the anonymous reviewers. This work has been partially funded by the German Federal Ministry of Education and Research (under grant titles 03IHS075A\&B) and by the German Federal Ministry for Economic Affairs and Energy (under grant title 01MF1170113D).

\section{References}

Conrad, J. et al. (2008), "What is design knowledge from the viewpoint of CPM/PDD?", Proceedings of the DESIGN 2008 / 10th International Design Conference, Dubrovnik, Croatia, May 19-22, 2008, The Design Society, Glasgow, pp. 745-752.

Conrad, J. et al. (2018), "Design Theory and Methodology in HCI: Applying CPM/PDD to UCD”, In: Marcus, A. and Wang, W. (Eds.), Design, User Experience, and Usability: Theory and Practice. 7th International Conference, DUXU 2018, Held as Part of HCI International 2018, Las Vegas, NV, USA, July 15-20, 2018, Proceedings, Part I (LNCS 10918), Springer, Cham, pp. 27-39. http://dx.doi.org/10.1007/978-3-319-91797-9_3

Dorst, K. (2011), “The core of 'design thinking' and its application”, Design Studies, Vol. 32, pp. 521-532. http://dx.doi.org/10.1016/j.destud.2011.07.006

d.school (2013), bootcamp bootleg. [online] Stanford University. Available at: https://dschool.stanford. edu/resources/the-bootcamp-bootleg (14-12-2018)

Eigner, M., Dickopf, T. and Huwig, C. (2016), "An interdisciplinary model-based design approach for developing cybertronic systems", Proceedings of the DESIGN 2016/14th International Design Conference, Dubrovnik, Croatia, May 16-19, 2016, The Design Society, Glasgow, pp. 1647-1656.

Gero, J.S. (1990), "Design prototypes - a knowledge representation schema for design", AI Magazine, Vol. 11 No. 4, pp. 26-36. 
Gothelf, J. and Seiden, J. (2012), Lean UX: Applying Lean Principles to Improve User Experience, O'Reilly, Beijing.

Haberfellner, R. et al. (2015), Systems Engineering - Grundlagen und Anwendung (Foundations and Applications), Orell Füssli Verlag, Zurich.

ISO (2018), ISO 9241-210: Ergonomics of human-system interaction. Part 210: Human-centered design process for interactive systems, ISO, Geneva.

Luedeke, T.F. et al. (2018), "CPM/PDD as an integrated product and process model for a design-thinking based, agile product development process", Proceedings of the DESIGN 2018/15th International Design Conference, Dubrovnik, Croatia, May 21-24, 2018, The Design Society, Glasgow, pp. 2063-2074. http://dx.doi.org/10.21278/idc.2018.0311

Pahl, G., Beitz, W. (2007), Engineering Design, Springer, Heidelberg. http://dx.doi.org/10.1007/978-1-84628$319-2$

Schön, D.A. (1983). The reflective practitioner: How professionals think in action, Temple Smith, London.

Steimle, T. and Wallach, D. (2018), Collaborative UX Design, dpunkt.verlag, New York.

Suh, N.P. (1990), The Principles of Design, Oxford University Press, New York.

Tafvizi Zavareh, M. et al. (2018), "A study on the socio-technical aspects of digitization technologies for future integrated engineering work systems", Proceedings of NordDesign 2018, Linköping, Sweden, August 1417, 2018, The Design Society, Glasgow.

Tullis, T. and Albert, B. (2013), Measuring the User Experience. Collecting, Analyzing, and Presenting Usability Metrics, 2nd ed., Morgan Kaufman/Elsevier, Waltham/MA.

VDI (1987), VDI-Guideline 2221: Systematic Approach to the Design of Technical Systems and Products, VDI, Düsseldorf, 1987.

Wallach, D., Conrad, J. and Steimle, T. (2017), "The UX metrics table: A missing artifact", In: Marcus, A. and Wang, W. (Eds.), Design, User Experience, and Usability: Theory, Methodology, and Management 6th International Conference, DUXU 2017, Held as Part of HCI International 2017, Vancouver, BC, Canada, July 9-14, 2017, Proceedings, Part I (LNCS 10288), Springer, Cham, pp. 507-517. http://dx.doi.org/ 10.1007/978-3-319-58634-2_37

Weber, C. (2005), "CPM/PDD - an extended theoretical approach to modelling products and product development processes", Proceedings of the 2nd German-Israeli Symposium on Advances in Methods and Systems for Development of Products and Processes, Berlin, Germany, July 7-8, 2005, Fraunhofer-IRBVerlag, Stuttgart, pp. 159-179.

Weber, C. (2014), "Modelling products and product development based on characteristics and properties", In: Chakrabarti, A. and Blessing, L.T.M. (Eds.), An Anthology of Theories and Models of Design. Philosophy, Approaches and Empirical Explorations, Springer, London, pp. 197-217. http://dx.doi.org/10.1007/978-14471-6338-1_16

Whitbeck, C. (1998), Ethics in Engineering Practice and Research, Cambridge University Press, Cambridge. 